\title{
Framework for Color and Texture Feature Fusion in Content Based Image Retrieval using Block Truncation Coding with Color Spaces
}

\author{
Sudeep D. Thepade, Rohan Awhad, Prakhar Khandelwal
}

\begin{abstract}
With tremendous growth in social media and digital technologies, generation, storing and transfer of huge amount of information over the internet is on the rise. Images or visual mode of communication have been prevailing and widely accepted as a mode of communication since ages. And with the growth of internet, the rate at which images are generated is growing exponentially. But the methods used to retrieve images are still very slow and inefficient, compared to the rate of increase in image databases. To cope up with this explosive increase in images, this information age has seen huge research advancement in Content Based Image Retrieval (CBIR). CBIR systems provide a way of utilizing the 3 major ways in which content is portrayed in images, those are shape, texture and color. In CBIR system, features are extracted from query image and similarity is found with features stored in database for retrieval. This provides an objective way of image retrieval, which is more efficient compared to subjective human annotation. Application specific CBIR systems have been developed and perform really well, but Generic CBIR systems are still under developed.

Block Truncation Coding (BTC) has been chosen as a feature extractor. BTC applied directly on input image provides color content-based features of image and BTC applied after applying $L B P$ on the image provide texture content-based features of image. Previous work consists of either color, shape or texture, but usage of more than one descriptor is still in research and might give better performance. The paper presents framework for color and texture feature fusion in content-based image retrieval using block truncation coding with color spaces.

Experimentation is carried out on Wang Dataset of 1000 images consisting of 10 classes. Each class has 100 images in it. Obtained results have shown performance improvement using fusion of BTC extracted color features and texture features extracted with BTC applied on Local Binary Patterns (LBP). Conversion of color space from RGB to LUV is done using Kekre's LUV.
\end{abstract}

Keywords: BTC, CBIR, Kekre's LUV, Wang Dataset.

\section{INTRODUCTION}

In today's Information age, images have become a common mode of communication over the internet. Capturing, storing and transferring image has become very easy, but retrieval of these images is still very inefficient. Image database is exploding with images and rate of image generation is growing exponentially but the development of

Revised Manuscript Received on February 05, 2020.

Sudeep D. Thepade*, Department of Computer Engineering, Pimpri Chinchwad College of Engineering, Pune, India.

E-mail: sudeepthepade@gmail.com

Rohan Awhad, Department of Computer Engineering, Pimpri Chinchwad College of Engineering, Pune, India.

E-mail: rohanawhad@gmail.com

Prakhar Khandelwal, Department of Computer Engineering, Pimpri Chinchwad College of Engineering, Pune, India.

E-mail: khandelwalprakhar30@gmail.com image retrieval systems is still very slow when compared to the rate of images generated.

There are two types of Image Retrieval systems based on text description and based on image content [1]-[5]. Text based Image Retrieval is done using keywords and metadata. Humans manually annotating large image database with keywords and metadata is very time consuming. Also, the assessment for efficacy of a keyword is undefined and subjective.

To retrieve images in an objective manner, Image Retrieval systems based on Image Content are used and they are standard across the entire database, unlike manually annotating image database, which is done using more than one human being. Images are rich in content. Content in an image is expressed in color, shape and texture. Individual consideration of either color [6], shape or texture [7] for generic retrieval systems is done in previous attempts, but the joint consideration [8] of more than one type of descriptors may boost the performance of image retrieval systems.

Application Specific Retrieval Systems are proposed, but for generic retrieval systems are not much explored. This paper proposes a fusion framework for CBIR using BTC applied on color spaces [9] and on local binary patterns [10].

Following represents structure of remaining paper. Section II explains the proposed fusion framework for CBIR system, section III contains BTC explanation and formulas, section IV explains LBP, section V contains information about the environment in which experiment is conducted, section VI explains result and observation of the experiments and finally section VII provides a conclusion for this experiment.

\section{PROPOSED FUSION FRAMEWORK FOR CONTENT BASED IMAGE RETRIEVAL}

Proposed CBIR framework is envisioned in 2 steps as extraction of features and image retrieval.

\section{A. Feature Extraction in proposed fusion based CBIR}

The color space of the Images from the database is converted from RGB color space to Kekre's LUV color space [11] and YCbCr color space [12]. These images go through a feature extraction process, which provides a vector of features for every image. The features are extracted by applying Block Truncation Coding (BTC) first time on color planes to get color features and to get texture features BTC is applied second time on Local Binary Patterns (LBP). These vectors of features for all images are stored in database.

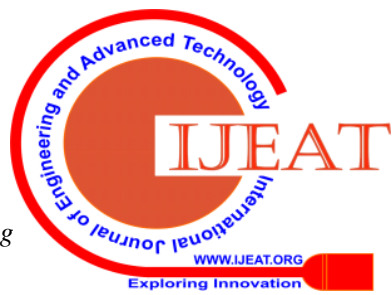


These feature vectors provide a way of comparison between images for retrieval purposes. In the proposed system, feature vectors of BTC on color planes and BTC on LBP patterns are concatenated together to get combined feature vector. Fig. 1 shows the step-by-step diagram for extraction of features for proposed system.

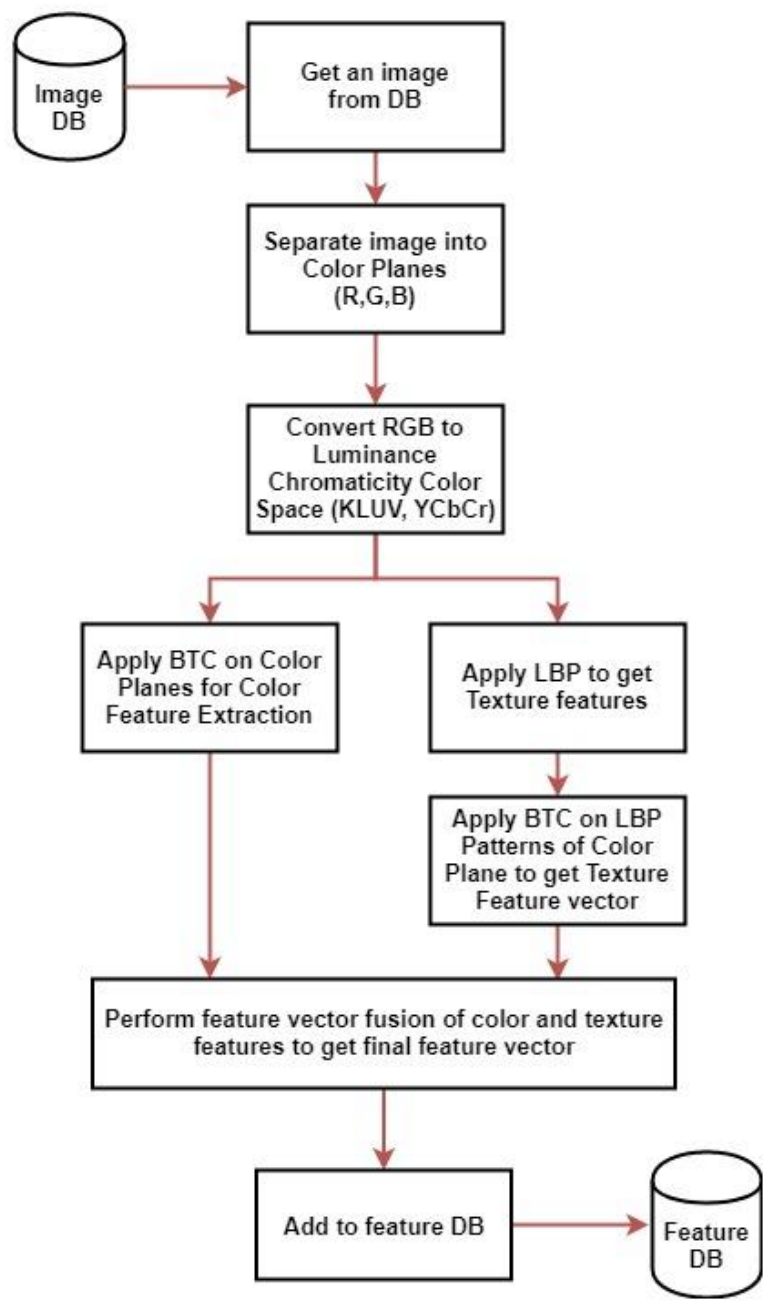

Fig. 1. Feature Extraction flow chart of proposed fusion framework for CBIR.

\section{B. Image Retrieval in proposed fusion based CBIR}

After getting a vector of features for query image as fusion of color features obtained with BTC applied on color planes of query image and texture features obtained with BTC applied on LBP patterns of color planes of query image, it is then compared to every other vector of image features stored in database. The comparison of images is done using Mean Squared Error (MSE) formula to get similarity percentage (0 being completely similar). Then images are sorted in ascending order using similarity percentage as key and first 100 images are retrieved. Fig. 2 shows image retrieval step-by-step diagram of the system proposed.

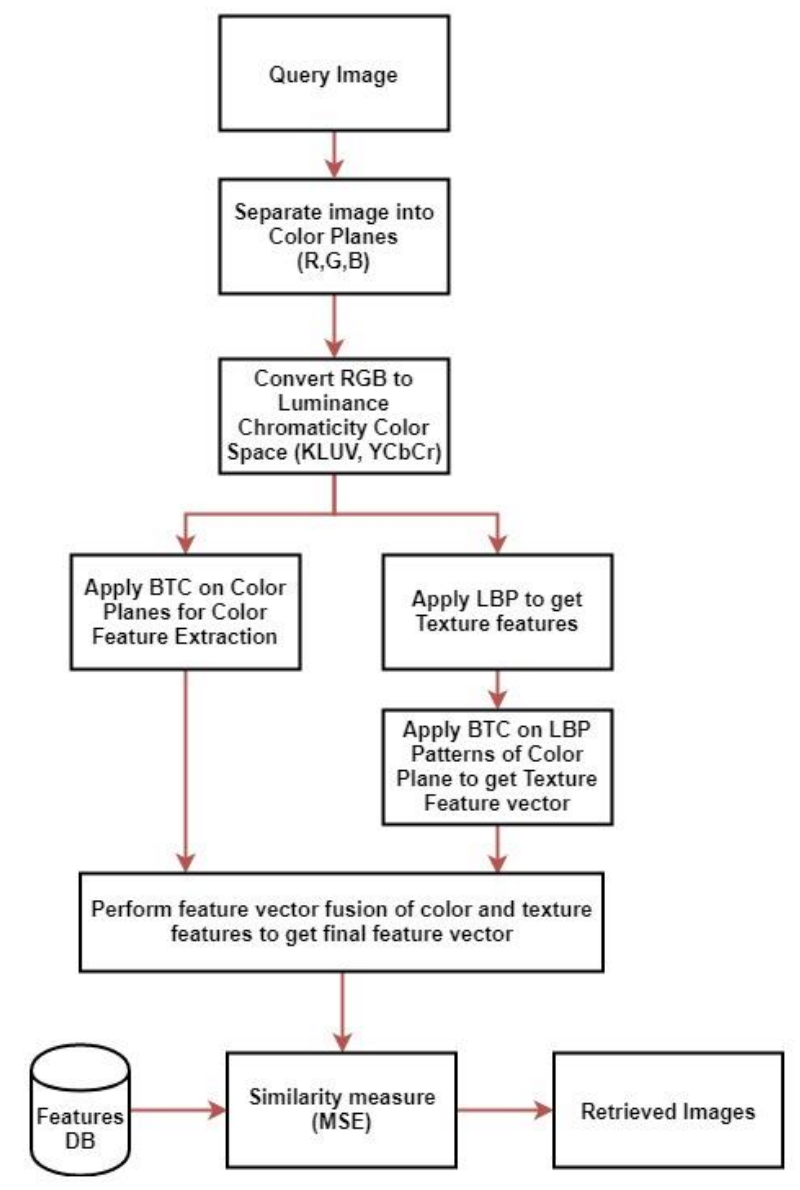

Fig. 2. Image Retrieval step-by-step diagram of proposed fusion framework for content-based image retrieval.

\section{BLOCK TRUNCATION CODING [13]}

Block Truncation Coding (BTC) is a technique to compress image. It was first envisioned for grayscale images, but it was quite innate to expand its scope to color images. The technique separates image into B, G and R components. For each component an independent threshold is calculated by taking mean of all pixel values for that component.

For explanation let's take $\mathrm{R}$ as Red plane first. Then array of pixel values, for $\mathrm{R}$ plane, is divided into 2 parts. First part consists of values less than the threshold for $\mathrm{R}$ and second part consists of values equivalent to or larger than threshold. The mean of the first part of the array is calculated and stored as a feature with name as LowerR and mean of second part is stored as UpperR. Similarly, LowerG, UpperG, LowerB and UpperB are calculated. These lower and upper values of all components are concatenated together, which represents BTC Level-1 feature vector consisting a total of 6 values for that image.

$$
\text { Threshold } R=\frac{1}{r * p} \sum_{a=1}^{a=r} \sum_{k=1}^{k=p} R(a, k)
$$




$$
\begin{aligned}
& \text { ThresholdG }=\frac{1}{r * p} \sum_{a=1}^{a=r} \sum_{k=1}^{k=p} G(a, k) \\
& \text { Threshold } B=\frac{1}{r * p} \sum_{a=1}^{a=r} \sum_{k=1}^{k=p} B(a, k)
\end{aligned}
$$

$$
\begin{aligned}
\text { Lower } R=\frac{1}{r * p} \sum_{\substack{a=1 \\
\text { if } R(a, k)<\text { Threshold } R}}^{a=r} \sum_{\substack{k=1 \\
k=p}} R(a, k),
\end{aligned}
$$

$$
\begin{aligned}
& \text { UpperR }=\frac{1}{r * p} \sum_{a=1}^{a=r} \sum_{k=1}^{k=p} R(a, k) \text {, } \\
& \text { if } R(a, k) \geq \text { ThresholdR }
\end{aligned}
$$

$$
\begin{aligned}
\text { Lower } G=\frac{1}{r * p} \sum_{\substack{a=1 \\
\text { if } G(a, k)<\text { Threshold } G}}^{a=r} \sum_{\substack{k=1 \\
k=p}} G(a, k),
\end{aligned}
$$

$$
\begin{aligned}
\text { Upper } G=\frac{1}{r * p} \sum_{\substack{a=1 \\
\text { if } G(a, k) \geq \text { Threshold } G}}^{a=r} \sum_{k=1}^{k=p} G(a, k)
\end{aligned}
$$

$$
\begin{aligned}
\text { Lower } B=\frac{1}{r * p} \sum_{\substack{a=1 \\
\text { if } B(a, k)<\text { Threshold } B}}^{a=r} \sum_{\substack{k=p \\
k}}^{k} B(a, k),
\end{aligned}
$$

For Multilevel-BTC, the first half array of every component is divided recursively using their Lower as threshold and second half using their Upper as threshold, until level has been reached.

In this experiment, Multilevel BTC was applied on 3 color spaces, namely RGB, LUV and YCbCr, with levels ranging from 1 to 4. Highest accuracy was observed for BTC Level-3 applied on LUV color space.

\section{LOCAL BINARY PATTERNS}

Local Binary Patterns or LBP is visual texture descriptor. In 1994, it was introduced, but stills proves to be a powerful texture feature classifier. LBP's compute a regional description of texture. This regional description is built by comparison of every pixel with its adjoining pixels. It is generally used for grayscale images, but it can be applied on individual image color components as a preprocessing step. For each pixel in a component, adjoining the center pixel, select a region of size ' $\mathrm{C}$ '.

For example, with $c=1$, a $3 \times 3$ locality of pixels is used by LBP descriptor. From this, take the center pixel and use it as a threshold for comparison with its neighbor. If the center pixel value is greater than neighboring pixel than write " 0 " else write "1". This is performed for all its 8 neighbors and order the neighbors in clockwise or counter-clockwise manner. Now, convert the 8-digit binary number, got from thresholding center pixel with 8 neighboring pixels, in a decimal number and place this value in output LBP image at the location of center pixel.

Two things which remain constant while determining LBP values is the starting neighbor and direction (clockwise or counter-clockwise) followed for the comparison of neighbors. This procedure of thresholding, collecting binary numbers and keeping the output decimal value in LBP image is replicated for every pixel in selected component. This is followed for all the components. It can be applied on different color space components, too. Experiment was performed by applying Multilevel BTC on LBP patterns, which was applied on individual components of a LUV color space image.

\section{EXPERIMENT ENVIRONMENT}

Experimentation is carried out on Wang Dataset [14] of 1000 images. Dataset consisted of 10 generic classes. 100 images per class. Experimentation is done in Python. Similarity between images is calculated using Mean Squared Error (MSE). In MSE, 0 represents completely similar and 100 represents completely different. Images are sorted in ascending order of their MSE value and top 100 are retrieved. Count of images belonging to query image's class are recorded. Accuracy of retrieval method is calculated by taking ratio of count of relevant retrieved images and count of all the images retrieved (i.e. 100). This process is carried out for all 1000 images as query image and average accuracy is calculated.

$$
M S E=\frac{\sum_{k=1}^{N}\left(y_{k}-\widehat{y_{k}}\right)^{2}}{N}
$$

where $y_{k} \& \widehat{y_{k}}$ denote the element at $\mathrm{k}^{\text {th }}$ index in the vector of image features. $\mathrm{N}$ denotes total count of all elements in vector of features. 
Framework for Color and Texture Feature Fusion in Content Based Image Retrieval using Block Truncation Coding with Color Spaces

$$
\text { Accuracy }=\frac{\begin{array}{c}
\text { Number of relevant } \\
\text { images retrieved }
\end{array}}{\begin{array}{l}
\text { Total number of } \\
\text { images retrieved }
\end{array}}
$$

\section{RESULTS AND DISCUSSION}

The experimentation is done for the CBIR using BTC for color features extraction, the CBIR with texture features extracted with BTC applied on Local Binary Patterns (LBP) of images and also the proposed CBIR with fusion of color \& texture features.

\section{A. CBIR with color features using Multilevel BTC}

Here the Multilevel BTC is applied on 3 color spaces, RGB,

LUV, and YCbCr with BTC levels ranging from 1 to 4 .

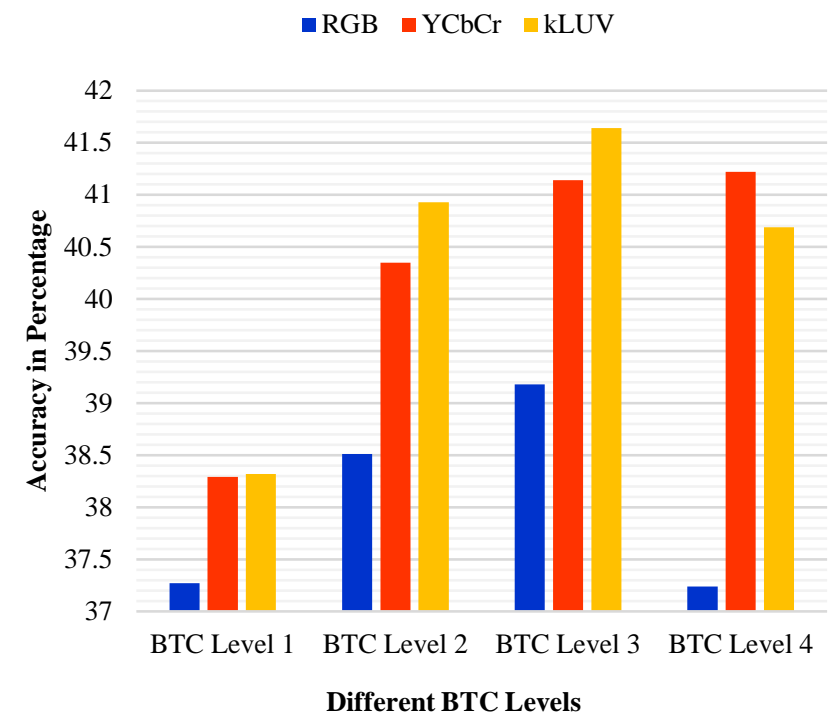

Fig. 3. Performance comparison of variations of color spaces in CBIR with color features averaged over respective BTC levels

Fig. 3 shows average retrieval accuracy comparison for the considered BTC levels with respective color spaces. It has been observed that LUV has given best performance among the considered color spaces from BTC level 1 to 3. In BTC level 4 YCbCr color space has performed better than LUV. BTC level 3 applied on LUV color plane has given best average retrieval accuracy of $41.64 \%$ for the generic image dataset.

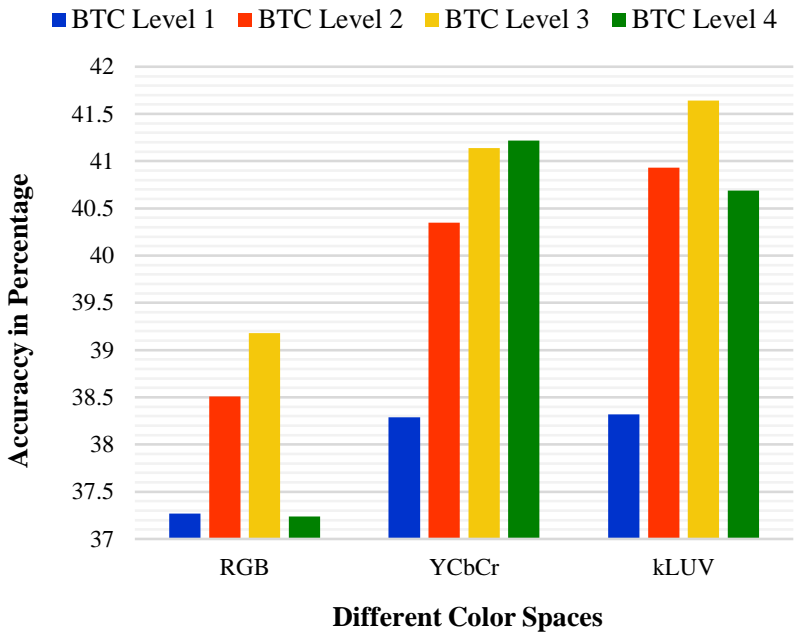

Fig. 4. Performance comparison of variations of BTC levels considered in proposed CBIR averaged over respective color spaces

Fig. 4 shows that average retrieval accuracy for 3 color spaces. Performance of BTC applied on RGB and LUV increases till Level 3 and then starts decreasing. BGR color space performs inferior when matched against YCbCr and LUV color spaces for all levels.

Table- I: Top 3 performers for BTC color feature based CBIR

\begin{tabular}{|c|c|c|c|}
\hline Rank & Level & Color Plane & $\begin{array}{c}\text { Percentage } \\
\text { Accuracy }\end{array}$ \\
\hline 1 & 3 & LUV & 41.64 \\
\hline 2 & 4 & YCbCr & 41.22 \\
\hline 3 & 3 & YCbCr & 41.14 \\
\hline
\end{tabular}

Table- I shows the top 3 performing variations of CBIR with color features using Multilevel BTC.

\section{B. CBIR using texture features extracted with Multilevel BTC applied on LBP Patterns}

In the experiment, Multilevel BTC is applied after, applying LBP on LUV and RGB color planes, with BTC levels ranging from 1 to 4 .

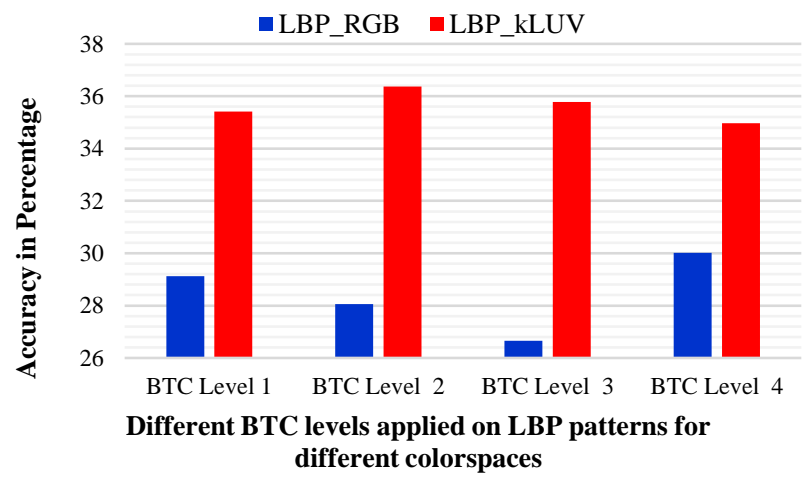

Fig. 5. Performance comparison of variations of LBP patterns applied over different color spaces in proposed CBIR with texture features averaged over respective BTC levels 
Fig. 5 shows average retrieval accuracy of 4 BTC levels applied on LBP patterns of respective color spaces. Performance for LUV increases till level 2 and then starts decreasing, whereas, performance for RGB color space decreases and then picks up after level 3. It has been observed that BTC applied on output of LBP patterns show a different trend for increasing levels when compared to BTC applied directly on color planes. BTC Level 2 applied on LUV LBP patterns performs the best with a $36.676 \%$ accuracy.

Table- II: Top 3 performers for texture features

\begin{tabular}{|c|c|c|c|}
\hline Rank & Level & Color Plane & $\begin{array}{c}\text { Percentage } \\
\text { Accuracy }\end{array}$ \\
\hline 1 & 2 & LUV & 36.376 \\
\hline 2 & 3 & LUV & 35.78 \\
\hline 3 & 1 & LUV & 35.416 \\
\hline
\end{tabular}

Table- II gives the top 3 performing variations of CBIR using texture features.

\section{Fusion of color features from Multilevel BTC and texture features from Multilevel BTC applied on LBP Patterns}

In the experiment, features of 3 best performing methods of CBIR with color features with BTC are concatenated with features of 3 best performing methods of CBIR with texture features with BTC applied on LBP patterns.

Table- III: Top 3 performers of feature fusion based CBIR variations.

\begin{tabular}{|c|c|c|c|c|c|}
\hline \multirow{2}{*}{ Rank } & \multicolumn{2}{|c|}{$\begin{array}{c}\text { Color Features } \\
\text { Method }\end{array}$} & \multicolumn{2}{|c|}{$\begin{array}{c}\text { Texture Feature } \\
\text { Method }\end{array}$} & \multirow{2}{*}{$\begin{array}{c}\text { Percentage } \\
\text { Accuracy }\end{array}$} \\
\cline { 2 - 5 } & Level & $\begin{array}{l}\text { Color } \\
\text { Plane }\end{array}$ & Level & Color Plane & \\
\hline 1 & 3 & LUV & 2 & LUV & 42.644 \\
\hline 2 & 4 & YCbCr & 3 & LUV & 40.062 \\
\hline 3 & 3 & YCbCr & 1 & LUV & 41.895 \\
\hline
\end{tabular}

Table- III provides top 3 performers of proposed feature fusion based CBIR.

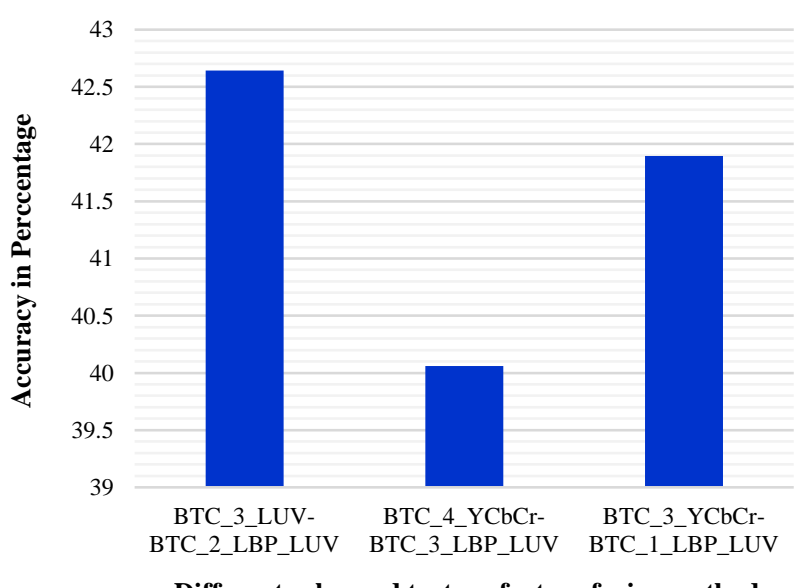

Fig. 6. Performance comparison of variations of proposed fusion of color and texture features in CBIR.
Fig. 6 shows average retrieval accuracy of 3 fusion methods. It has been observed that this type of feature fusion led to an increase in accuracy. It has been observed that features of BTC applied on LBP patterns, which are calculated from LUV color planes when concatenated with features of BTC applied directly on LUV color plane gives better accuracy when compared to other fusion methods. Fusion of BTC Level 3 applied on LUV color plane and BTC Level 2 applied on LBP patterns of LUV color plane performs the best with a $42.644 \%$ accuracy.

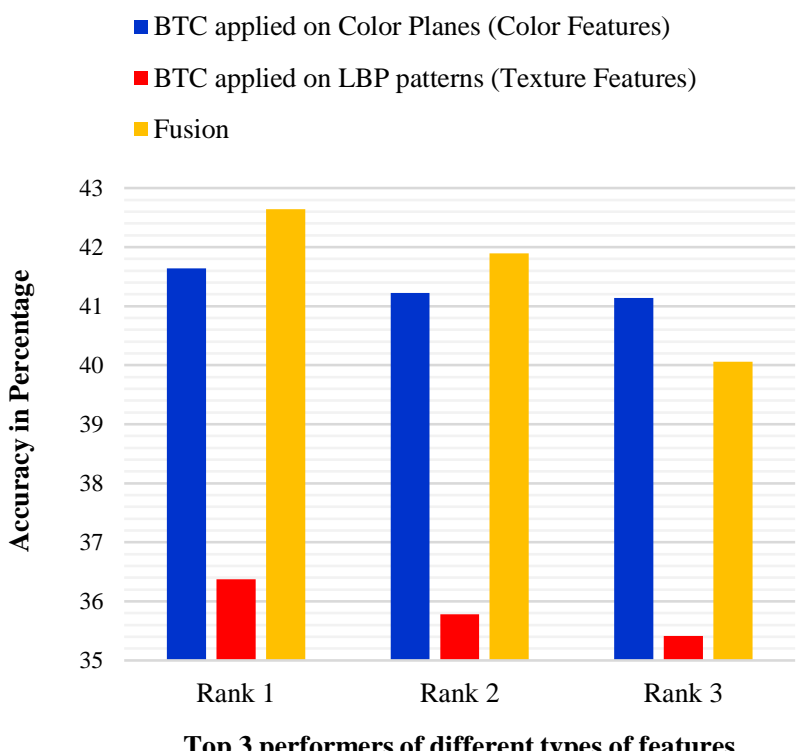

Fig. 7. Performance comparison of top 3 methods of color features, texture features and their proposed fusion in CBIR.

Fig. 7 shows performance comparison of top 3 performers of BTC applied on color planes, BTC applied on LBP patterns and their fusion. It has been observed BTC applied directly on color spaces gives better accuracy when compared to BTC applied on LBP patterns. Color-texture feature fusion gives the best accuracy among only color or only texture features.

\section{CONCLUSION}

In today's age where capturing and storing of images has grown exponentially, retrieval of those images is still based on annotations given by human, which is subjective and has errors. Therefore, CBIR gives a way of image retrieval based solely on features which are objective, and hence more accurate. Image content is exhibited in three features, namely color, shape and texture. The paper proposes fusion framework for color and texture features calculated using Multilevel BTC applied on color planes and on LBP patterns. Experimentation is performed on Wang dataset of 1000 images with 10 classes. Multilevel BTC applied directly on color planes increases accuracy up to level 3 and after that stabilizes. Whereas, Multilevel BTC applied on LBP patterns, increases accuracy up to level 2 and then starts decreasing.

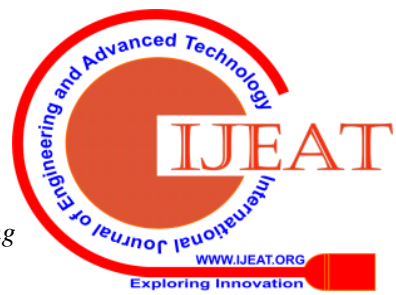


It is observed that Multilevel BTC applied on color planes and Multilevel BTC applied on LBP patterns show different trend for increasing level. The fusion of BTC features for color and texture content give the best results, with combination of BTC Level 3 applied on LUV color planes and BTC Level 2 applied on LBP patterns, which were calculated on LUV color plane. The proposed fusion of CBIR with color \& texture features is given performance boost in retrieval accuracy.

\section{REFERENCES}

1. Ritendra Datta, Jia Li, James Z. Wang, "Content-based image retrieval approaches and trends of the new age",

2. A. W. M. Smeulders, M. Worring, S. Santini, A. Gupta, and R. Jain, "Content-based image retrieval at the end of the early years", IEEE Trans. Pattern Anal. Mach. Intell., vol. 22, no.12, December 2000, pp. 1349-1380.

3. M. Kokare, B. N. Chatterji, and P. K. Biswas, "A survey on current content-based image retrieval methods", IETE J. Res., vol. 48, no. 3\&4, 2002, pp. 261-271.

4. Y. Liu, D. Zhang, G. Lu, and W.-Y. Ma, "A survey of content-based image retrieval with high level semantics", Pattern Recogn., vol. 40, no. 1, January 2007, pp. 262-282.

5. Y. Rui and T. S. Huang, "Image retrieval: current techniques, promising directions and open issues", J. Visual Commun. Image Represent., vol. 10, no. 1, March 1999, pp. 39-62.

6. Dr. H. B. Kekre, Dr. Sudeep D. Thepade, and Akshay Maloo, "Query by image content using colour averaging techniques", International Journal of Engineering Science and Technology, vol. 2, no. 6, 2010, pp. 1612-1622.

7. B. S. Manjunath and W. Y. Ma, "Texture features for browsing and retrieval of image data", IEEE Transactions Pattern Anal. Mach. Intell., vol. 18, no. 8, August 1996, pp. 837-842.

8. M. Subhrahmanyam, A. B. Gonde, and R. P. Maheshwari, "Color and texture features for image indexing and retrieval”, in Proc. IACC, 2009, pp. 1411-1416.

9. Guoping Qiu, "Color image indexing using btc", IEEE Transactions on Image Processing, January 2003, VOL. 12. NO. 1, pp. 93-101.

10. T. Ojala, M. Pietikainen, and T. Maenpaa, "Multiresolution gray-scale and rotation invariant texture classification with local binary patterns", IEEE Trans. Pattern Anal. Mach. Intell., vol. 24, no. 7, July 2002, pp.971-987.

11. Dr. H. B. Kekre, Dr. Sudeep D. Thepade, "Boosting block truncation coding using Kekre's LUV color space for image retrieval", WASET International Journal of Electrical, Computer and System Engineering (IJECSE), Volume 2, Number 3, Summer 2008, pp. 172-180.

12. Dr. H. B. Kekre, Dr. Sudeep D. Thepade, "Color based image retrieval using amendment block truncation coding with $\mathrm{YCbCr}$ color space", International Journal on Imaging (IJI), Volume 2, Number A09, Autumn 2009, pp. 2-14. Available online at www.ceser.res.in/iji.html (ISSN: 0974-0627).

13. Dr. H. B. Kekre, Dr. Sudeep D. Thepade, Shrikant P. Sanas, "Improved CBIR using multileveled block truncation coding", International Journal on Computer Science and Engineering, Vol. 02, No. 07, 2010, pp. 2471-2476.

14. James Z. Wang, http://wang.ist.psu.edu/ jwang/test1.tar (Last Referred on 19 December 2019), September 2008. http://wang.ist.psu.edu/

\section{AUTHORS PROFILE}

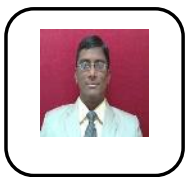

Sudeep D. Thepade is currently Professor in Computer Engineering Department at Pimpri Chinchwad College of Engineering affiliated to Savitribai Phule Pune University, Pune, Maharashtra, India. He has completed Ph.D. in 2011. He has more than 350 research papers to his credit published in International/ National Conferences and Journals. His domain of interest is Image Processing, Image Retrieval, Video Analysis, Video Visual Data Summarization, Biometrics and Biometric Liveness Detection. He is member of International Association of Engineers (IAENG) and International Association of Computer Science and Information Technology (IACSIT). He has served as Technical Program Committee member and Reviewer for Several International Conferences and Journals.

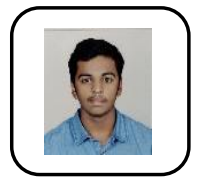

Rohan Awhad is currently pursuing B. E. (Computer Engineering) from Pimpri Chinchwad College of Engineering affiliated to Savitribai Phule Pune University, Pune. His areas of interest are Deep Learning, Machine Learning, Cyber Security, Data Science, Full Stack Development and Film-making. He has several projects in data science, web development, software development such as SLAM, Chess engine built using Deep Reinforcement Learning, Mailing System for government, Automated data entry system. He also has a video on YouTube about Amazon's strategies and growth. He has competed in various intercollegiate programming competitions. He has also participated in Kaggle competitions. He is currently working under Dr. Sudeep D. Thepade.

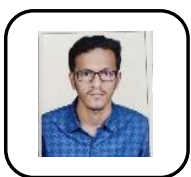

Prakhar Khandelwal is currently pursuing B. E. (Computer Engineering) from Pimpri Chinchwad College of Engineering, Savitribai Phule Pune University, Pune, Maharashtra, India. His areas of interest are Data Science and Backend Development. He has completed his internship at Walnut (money manager and instant personal loans app) as a Software Developer. He has multiple projects in Data Science and Backend Development. He has completed workshop on Applied CS with Android by Google and was nominated for Google Student Facilitator for the same. Ranked 6th out of 50 teams in Pune Coding League (PCL 3.0) which was conducted by PCCOE ACM Student Chapter. He also conducted sessions on Machine Learning for his college students under PCCOE ACM Student Chapter. He is currently working under Dr. Sudeep D. Thepade. 